\title{
Philosophiques
}

\section{Daubert et les limites de la phénoménologie : Étude sur le donné et l'évidence}

\section{Guillaume Fréchette}

Volume 28, numéro 2, automne 2001

URI : https://id.erudit.org/iderudit/005667ar

DOI : https://doi.org/10.7202/005667ar

Aller au sommaire du numéro

Éditeur(s)

Société de philosophie du Québec

ISSN

0316-2923 (imprimé)

1492-1391 (numérique)

Découvrir la revue

Citer cet article

Fréchette, G. (2001). Daubert et les limites de la phénoménologie : Étude sur le donné et l'évidence. Philosophiques, 28(2), 303-326.

https://doi.org/10.7202/005667ar
Résumé de l'article

Johannes Daubert (1887-1947) est la figure centrale du Cercle de Munich ainsi que le premier véritable lecteur et critique de Husserl. Ses manuscrits contiennent, en plus d'une critique de la phénoménologie husserlienne, une conception originale de la phénoménologie laissant notamment une place importante aux analyses perceptives. Le présent article s'intéresse d'abord aux thèmes du donné ( gegeben, Selbstgegeben ) et de l'évidence en tant qu'ils sont des motifs centraux à la fois chez Husserl et Daubert, pour ensuite relever, à partir d'une étude des manuscrits pertinents, la particularité des analyses daubertiennes concernant ces thèmes, ainsi que les contraintes ou limites que la phénoménologie doit s'imposer pour notamment se distinguer du rationalisme. 


\title{
Daubert et les limites de la phénoménologie : Étude sur le donné et l'évidence'
}

\author{
GUILLAUME FRÉCHETTE \\ Universität Hamburg \\ guillaume.frechette@public.uni-hamburg.de
}

\begin{abstract}
RÉSUMÉ. - Johannes Daubert (1887-1947) est la figure centrale du Cercle de Munich ainsi que le premier véritable lecteur et critique de Husserl. Ses manuscrits contiennent, en plus d'une critique de la phénoménologie husserlienne, une conception originale de la phénoménologie laissant notamment une place importante aux analyses perceptives. Le présent article s'intéresse d'abord aux thèmes du donné (gegeben, Selbstgegeben) et de l'évidence en tant qu'ils sont des motifs centraux à la fois chez Husserl et Daubert, pour ensuite relever, à partir d'une étude des manuscrits pertinents, la particularité des analyses daubertiennes concernant ces thèmes, ainsi que les contraintes ou limites que la phénoménologie doit s'imposer pour notamment se distinguer du rationalisme.
\end{abstract}

\begin{abstract}
Johannes Daubert (1887-1947) is the central figure of the Munich Circle as well as the first real critical reader of Husserl. His manuscripts contain, in addition to a critique of the husserlian phenomenology, an original conception of phenomenology leaving a significant place to the perceptive analyses. This article is initially concerned with the topics of the given (gegeben, Selbstgegeben) and of the evidence as they are central themes in Husserl's and Daubert's works, for then raising, starting from a study of the relevant manuscripts, the peculiarity of the daubertian analyses concerning these topics, as well as the constraints or limits that the phenomenology must impose itself to be distinguished from the Rationalism.
\end{abstract}

\section{L'évidence et le donné dans la phénoménologie}

Qu'y a-t-il à comprendre par description ? Qu'est-ce que peut revendiquer une philosophie qui emploie la description comme méthode ? La phénoménologie, telle que définie par Husserl dans l'introduction à la première Recherche logique,

représente un domaine de recherches neutres, dans lequel les différentes sciences ont leurs racines. D'une part, elle sert à la préparation de la Psychologie en tant que science empirique. Elle analyse et décrit (spécialement en tant que phénoménologie du penser et du connaître) les vécus de représentation, de jugement, de

1. La présente étude est une version remaniée de l'article "Daubert e i limiti della fenomenologia : studio sul dato e l'evidenza " à paraître dans S. Besoli (dir.), Realismo fenomenologico. Elle a été rendue possible grâce à une bourse de recherche du Fonds pour les Chercheurs et l'Aide à la Recherche du Québec (FCAR) que je remercie. À un autre titre, je remercie également $\mathrm{K}$. Schuhmann qui m’a généreusement donné accès à ses transcriptions des manuscrits sténographiés de Daubert, qui sont à la base de la présente étude et sans lesquelles elle n'aurait pu être réalisée. 
connaissance, qui doivent trouver leur explication génétique ainsi que leur investigation selon des relations empirico-nomologiques dans la psychologie. D'autre part, elle révèle les "sources" d'où « découlent" les concepts fondamentaux et les lois idéales de la logique pure, et jusqu'auxquelles il faut les faire remonter pour leur procurer "la clarté et la distinction " nécessaires à une compréhension épistémologiquement critique de la logique pure ${ }^{2}$.

Ce passage dévoile les grandes lignes d'une définition de la phénoménologie en tant que psychologie descriptive, définition qui consiste en une tâche bipartite : d'une part, servir à la préparation de la psychologie comprise comme science empirique et, d'autre part, mettre à découvert les sources d'où jaillissent les concepts fondamentaux et les lois idéales de la logique pure, sources jusqu'auxquelles ceux-ci doivent être remontés, afin de leur procurer "clarté et distinction ». Ainsi, une pure phénoménologie se doit d'analyser et décrire de façon neutre les vécus de représentation, de jugement et de connaissance, qui doivent trouver leur explication génétique dans la psychologie, et elle doit à la fois procurer "clarté et distinction » aux concepts fondamentaux et aux lois idéales de la logique pure. Dans son analyse et sa description des vécus, elle s'applique à pratiquer ni plus ni moins qu'une psychologie descriptive, et dans sa tentative de procurer "clarté et distinction ", de " rendre évident » ce qui constitue la logique pure, elle cherche à en "dégager les sources ". Cette double tâche se manifestait déjà dans les Prolégomènes, notamment en discriminant toute théorie psychologique de la logique concernant les sources de l'évidence et la logique pure.

Plus généralement, la nécessité d'une permanente double tâche de la phénoménologie devient explicite lorsqu'on observe la manière par laquelle les thèmes directeurs de l'œuvre de Husserl seront développés. Par exemple, c'est le cas de la distinction polémique entre psychologie et logique, introduite entre autres dans le contexte d'une présence accrue de la psychologie dans les départements de philosophie allemands, où Husserl tente de définir la tâche philosophique de la psychologie. Cette double tâche se manifeste également plus tard par la distinction entre deux modes de phénoménologie, intersubjective et transcendantale, qui insistent respectivement sur l'occasionnalité du monde de la vie, de la Lebenswelt, et sur la fondation par la subjec-

2. «(...) stellt ein Gebiet neutraler Forschungen dar, in welchem verschiedene Wissenschaften ihre Wurzeln haben. Einerseits dient sie zur Vorbereitung der Psychologie als empirischer Wissenschaft. Sie analysiert und beschreibt (speziell als Phänomenologie des Denkens und Erkennens) die Vorstellungs-, Urteils-, Erkenntniserlebnisse, die in der Psychologie ihre genetische Erklärung, ihre Erforschung nach empirisch-gesetzlichen Zusammenhängen finden sollen. Andererseits erchließt sie die "Quellen“, aus denen die Grundbegriffe und die idealen Gesetze der reinen Logik „entspringen“, und bis zu welchen sie wieder zurückverfolgt werden müssen, um ihnen die für ein erkenntniskritisches Verständnis der reinen Logik erforderliche „Klarheit und Deutlichkeit“ zu verschaffen ». Hua XIX1/6-7 (notre traduction). Toutes les références aux textes de Husserl renvoient à la collection Husserliana (abrégé ici Hua), l'édition de son œuvre complète, par numéro de tome (XIX1) et de page (6-7). 
tivité, par le Je. Dans les deux cas, il semble que la double tâche mise de l'avant dans l'introduction à la première Recherche logique soit toujours présente : analyser et décrire les différents vécus, les différents modes de donations (perception, jugement, représentation) avec les instruments de la psychologie descriptive (plus tard développée comme psychologie phénoménologique) mais également procurer clarté et distinction aux fondements de la logique pure.

Ce double enjeu de la phénoménologie de Husserl n'a peut-être pas la continuité qu'il suggère, si l'on veut croire qu'avec la réduction est acquise une reconstitution continue, "absolue ", des vécus de la conscience dans un " flux » dont l'unité est dans un premier temps la conscience du temps, et dans un second la conscience pure ${ }^{3}$. En effet, si la réduction peut rassembler l'ensemble du flux des vécus de la conscience dans une "unité des vécus " ${ }^{4}$, elle doit d'autre part être en mesure de donner à cette unité un fondement d' "une toute autre nature » que la simple adéquation, et l'évidence obtenue alors est d'une toute autre valeur pour la connaissance. Que l'on accepte ou non un tel fondement transcendantal de l'évidence, celle-ci demeure toutefois «donnée », et cette corrélation avec ses modes de donation, qu'elle mette en jeu la problématique psychologique ou transcendantale, fait somme toute intervenir le même motif : le fait de la donation des objets.

La présente étude tentera d'examiner ce "fait de la donation des objets » dans la phénoménologie, en prenant assise sur certains manuscrits de Daubert qui traitent de la question. Dans les Recherches logiques, Husserl fait un usage des termes de donation (Gegebenheit), de donné-même (Selbstbegeben, Selbstgegebenheit $)^{5}$ qui renvoient essentiellement à une définition

3. Nous renvoyons ici à deux textes déterminants du corpus husserlien, respectivement les Texte zur Phänomenologie des inneren Zeitbewußtseins (Hua X/44sq.), et les Ideen... (Hua III/99sq. et 180 sq.).

4. Des expressions telles que unité des vécus (Erlebniseinheit), unité de conscience (Bewußtseineinheit) ou encore flux des vécus (Erlebnisstrom) sont des expressions typiques utilisées par Husserl pour décrire ce que réalise la réduction sur la conscience. Qui plus est, elle sont même directement utilisées dans l'édition révisée des Recherches logiques pour décrire, dans la sixième $R l$, un sens du mot phénomène (Erscheinung) : « ... en considérant que les vécus de toute sorte (...) peuvent aussi devenir les objets d'intuitions réflexives, internes, tous les vécus, dans l'unité des vécus d'un Je, se nomment alors des " phénomènes ". Dans cette mesure, la phénoménologie correspond, de façon générale, à la doctrine des vécus (...). La phénoménologie est alors la doctrine des essences des "phénomènes purs ", ceux de la " conscience pure », d'un "Je pur » » (Hua XIX2/765). Notre traduction.

5. Nous traduisons ici Selbstgegebenheit également par donné-même (plus précisément, le fait du donné-même), d'une part afin d'éviter les confusions que peut susciter le terme « autodonation » par son caractère trop indéterminé, sinon tautologique (voir Marion, 1997, pour une perspective différente sur la question de l'autodonation). D'autre part, nous traduisons ainsi parce que Husserl n'emploie pas ce terme dans la première édition des $R l$, où il parle tout au plus d'un donné " propre » (selbst gegeben) pour qualifier la représentation propre du général ( eigentümliche » Vorstellung des Allgemeinen) dans l'abstraction actuelle (cf. HuaXIX1/149). Nous introduisons ici ce terme en ce que Daubert en fait un usage dans plusieurs manuscrits, sur lesquels nous reviendrons plus loin. 
de l'évidence comme adéquation ou encore concordance (Adäquation, Übereinstimmung) (voir HuaXIX2/651-2) ${ }^{6}$. On peut dire que cette définition de l'évidence, en termes d'adéquation et de concordance, correspond pour l'essentiel au sens que donne Daubert à ce concept. Rappelons qu'il soutient, dans l'ensemble, une position assez proche de celle de Reinach en ce qui concerne la nature de la phénoménologie, qu'il serait juste de qualifier de réaliste, d'une part en ce qui a trait aux thèses ontologiques qu'ils soutiennent, et d'autre part en opposition à la seconde philosophie de Husserl, à caractère clairement idéaliste, développée dans les Ideen...7. Aussi, nous insisterons ici sur les critiques et les questions soulevées par Daubert eût égard à cette question de l'évidence et de ses modes de donation, qui constitue, comme nous l'avons suggéré plus haut, la double topique de la phénoménologie husserlienne. De par son refus à pratiquer toute forme de réduction phénoménologique, nous verrons par quels moyens Daubert conçoit délimiter ces concepts d'évidence et de donné ou donation, et quelles sont les « limites » qu'il impose à la phénoménologie ${ }^{8}$.

\section{Les déterminations possibles de la phénoménologie : les différents modes du donné.}

Dans son étude sur les déterminations possibles de la phénoménologie (Die möglichen Bestimmungen der Phänomenologie $)^{9}$, Daubert tente précisément de voir de quelle manière la phénoménologie, par son analyse et sa description, parvient à rendre son objet en toute "clarté et distinction ». Dans ce manuscrit qu'il n'a pas daté, Daubert présente une définition de la phénoménologie en sept volets, suivis d'une conclusion ${ }^{10}$. Ces déterminations se

6. Contrairement à la seconde édition, la première édition des $R l$ ne décrit l'évidence que très rarement en terme de donation ou de donné.

7. Sur la phénoménologie de Daubert, ainsi que sur ses écrits, voir Schuhmann, 1985, 1998 ; Schuhmann et Smith, 1985, 1987, 1991 ; Smid, 1985.

8. Pour une présentation historique de Daubert et du contexte de la phénoménologie de Munich, nous renvoyons aux articles cités dans la note précédente, ainsi qu'à Spiegelberg, 1982, pp. 166-239 ; Schapp, 1976 ; Kuhn, Avé-Lallemant, Gladiator (dir.), 1975 ; Sepp (dir.), 1988 ; Avé-Lallemant, 1975 ; Schuhmann, Avé-Lallemant, 1992 ; Spiegelberg, Avé-Lallemant (dir.), 1982. Pour une édition française de certains manuscrits de Daubert, voir Fréchette, 2000, pp. $149-170$.

9. Sur ce manuscrit, voir notamment Smid, 1982, pp.109-153.

10. À première vue, dans le corps du texte, Daubert décrit la phénoménologie de Husserl comme " rationaliste ", une description qui laisse entendre que le texte ait pu être écrit au moins autour des années 10. Cependant, comme nous le verrons dans les manuscrits qui suivent, Daubert adressait cette critique contre la phénoménologie de Husserl bien avant que celle-ci se manifeste explicitement rationaliste. D'autre part, par sa ressemblance importante avec un manuscrit de la Mappe 11 (A I 11/18), daté celui-ci de juillet 1906, il est justifié de croire qu'il fut rédigé la même année. Voir à cet effet Schuhmann, 1996. 
fondent pour la plupart dans la donation et le donné-même (Selbstgegebenheit), un concept récurrent dans les manuscrits de Daubert. Dans une discussion avec Pfänder sur le réalisme, Daubert affirmait, au sujet du réalisme critique : " (...) Que les donations soient subjectives, que le monde effectif, réel, soit posé à la base de ces donations, parce qu'il est évident que selon un contexte régulé un ordre puisse être supposé entre elles, sans lequel nous ne pourrions pas vivre $»^{11}$.

Les donations sont donc subjectives, elles embrassent l'ensemble des catégories entrant dans la composition du monde réel, dont l'ordre n'est pourtant pas subjectif. En ce sens, les donations doivent être entendues comme des phénomènes subjectifs comprenant de quelque façon sousjacente les règles d'ordonnancement entre les catégories.

La première détermination de la phénoménologie correspond à cette distinction entre le caractère subjectif des donations et leur ordonnancement nécessaire. Elle est celle de la

description des apparitions telles qu'elles ont une réalité primaire. Les objets tels qu'ils sont pensés avec toutes les illusions et les intentions impropres mélangés avec les donnés-mêmes propres. Lorsqu'on amène ces objets au donné-même, c'est alors un regard réfléchissant, non le même regard qui saisit les objets pertinents de la pensée. Or, et c'est là le problème, le regard réfléchissant présume une autre conscience-de immédiate parce qu'il ne peut être acquis de l'obscurité ${ }^{12}$.

Cette réalité primaire est celle de la donation comme phénomène subjectif au sens le plus commun du terme, ne distinguant aucunement les objets des illusions et des intentions impropres. Leur considération à titre de donné-

11. «(...) Daß die Gegebenheiten subjektiv sind, und die wirkliche, reale Welt diesen Gegebenheiten irgendwie zugrunde gelegt wird, weil sich herausstellt, daß sich nach einem bestimmten gesetzlichen Zusammenhang eine Ordnung zwischen ihnen anzunehmen ist, ohne die wir nicht leben könnten ». Discussion du 21 juin 1931 avec Pfänder, rapportée dans A I 3/ 5. Les manuscrits de Daubert, tels que Avé-Lallemant les reçût de la veuve de Daubert en 1967, sont composés de trois séries de cartables (Mappen) : A : notes et manuscrits ; B : Correspondance ; $\mathrm{C}:$ Personalia, d'où la notation de référence que nous utilisons, par exemple A I 1/4, le I signifiant la première section de la série A, le 1 le numéro du Mappe particulier et le 4, le numéro de folio. Cependant, l'ordonnancement personnel de Daubert a pu être dérangé par la fouille de sa ferme à Freidlhof par les troupes d'occupation américaines après la Seconde Guerre Mondiale, celles-ci ayant probablement douté de l'écriture sténographique de ces manuscrits (voir Avé-Lallemant, 1975, p. 128). Sur les manuscrits regroupés dans le Mappe A I 3, voir Schuhmann, 1989.

12. "Als Beschreibung der Erscheinungen so wie sie primäre Wirklichkeit haben. Die Gegenstände so wie sie gemeint sind mit allen Täuschungen und eingeflochtenen uneigentlichen Intentionen, vermischt mit den eigentlichen Selbstgegebenheiten. Wenn man dieses zur Selbstgegebenheit bringt, dann ist es ein reflektierender Blick, nicht der Blick, welcher selber die betreffenden Gegenstände der Meinung fasst. Das Problem ist nun dieses, dass der reflektierende Blick schon ein anderes unmittelbares Bewusstsein-von voraussetzt, weil er nicht aus dem Dunkel erstehen lassen kann ». 
même nécessite alors un regard différent du regard saisissant les objets. De cette façon, il serait nécessaire de supposer une seconde instance, une seconde conscience telle qu'elle sera proposée par Husserl dans les Ideen. Mais pour Daubert, la question de la donation fait d'abord intervenir celle de la saisie :

Ce qui, en tant que même, ce qui est pensé, est également donné (adéquation du donné et du saisi). Dès lors, le problème des différents modes de l'être-saisi et de l'être-donné. Par là, différentes sphères d'être. Bien qu'encore un problème ouvert, à savoir si les sphères d'être peuvent être déterminées seulement selon le mode de l'être-donné, ou encore comment entrent en jeu l'être-saisi et les modes d'évidences. C'est le sens propre du donné-même ${ }^{13}$.

Autrement dit, ce qui est pensé comme identique, à partir du regard " qui saisit les objets pertinents de la pensée », donc le regard usuel, est donné du même droit qu'il est visé ou pensé (gemeint), sans discrimination quant à d'autres modes de conscience ${ }^{14}$. Cependant, nous pouvons également dire que tout ce qui est pensé est déjà nécessairement donné : cette considération vient en outre appuyer celle selon laquelle les donations ont un double statut ; elles sont pour ainsi dire subjectives en ce qui concerne leur aspect épistémologique et elles sont objectives en ce qui a trait à leur ontologie. Aussi, le problème mis de l'avant avec cette deuxième détermination est le suivant : si nous considérons les donations comme étant toutes de même droit pour la connaissance, et que nous considérons parallèlement qu'elles sont adéquates au saisi, il faut donc supposer une ontologie parallèle du saisi et du donné, à quoi devraient correspondre différents modes ou " sphères » d'être. Le problème du donné-même sera précisément le suivant : peut-on directement déterminer une ontologie correspondante du donné et du saisi en partant seulement du donné ? Et comment sont réglés les modes d'évidence et du saisi ? Husserl répond à cette question dans Ideen ... en instituant la connaissance sur le donné, faisant ainsi de la donation le principe par lequel se fonde la phénoménologie constitutive ${ }^{15}$. Or, pour Daubert, la donation ne peut être atteinte qu'à partir du pensé, bien qu'elle garde d'un point de vue ontologique un statut semblable à celui que lui accorde Husserl dans l'ouvrage cité plus haut.

13. "Das, was als dasjenige, was es gemeint ist, auch gegeben ist (Adäquation von gegeben und erfasst). Hier das Problem der verschiedenen Arten des Bewusstseins und des Gegebenseins. Dadurch verschiedene Seinssphären. Wobei noch offenes Problem, ob die Seinssphären nach der Art des Gegebenseins allein bestimmt werden können oder wie das Erfasstsein und die Evidenzarten hereinspielen. Dieses der eigentliche Sinn des Selbstgegeben ».

14. La traduction de meinen par viser est généralement acceptée, mais comme sa signification en allemand est plus large que celle de la traduction française, nous employons parfois les termes de façon interchangeable.

15 . Voir notamment le $\$ 24$ des Ideen. 
La troisième détermination de la phénoménologie concerne l'objet de la donation :

Ce qui est donné en même, également lorsqu'il n'est pas pensé en lui-même. Ce qui a une réalité de donation. Par exemple, comme celle de la pensée des choses, etc., les modes de donations entretissés des couleurs, etc., même si cela n'est pas saisi en visant. La chose que le positivisme entend par pure donation. Mais un produit de l'abstraction. Ici encore, le problème de II- [de la deuxième détermination] selon les sphères d'être et les modes d'être-donné ${ }^{16}$.

Cette troisième détermination concerne l'objet de la donation, dans ce que le phénomène a d'essentiellement objectif. Cet objet peut être indépendant d'un objet de pensée, et donc compris comme objet au sens le plus large : la composition du rouge et du jaune telle qu'elle produit l'orange et telle que ce produit est pensé, perçu, constitue ce type d'objet ${ }^{17}$. Daubert introduit ici un parallèle important avec le positivisme : ce type d'objet privilégié par la phénoménologie est à comprendre comme ce qu'entend le positivisme comme pure donation. Ce que Daubert entend ici par positivisme correspond aux théories et aux méthodes développées entre autres par Avenarius et $\mathrm{Mach}^{18}$ : cette forme de positivisme, comme le remarque M. Sommer, a quelques similitudes avec la phénoménologie husserlienne :

ainsi, ce que cherchent communément Avenarius, Mach et Husserl : une nouvelle immédiateté aux choses mêmes ; une nouvelle fidélité aux choses dans la théorie ; une nouvelle compréhension de la relation subjective au monde ; une nouvelle sorte d'évidence ${ }^{19}$.

16. «Das, was als selbst gegeben ist, auch wenn es nicht selbst gemeint ist. Das, was Gegebenheitsrealität hat. z.B. wie der Meinung von Dingen usw., die eingewobenen Gegebenheitsarten der Farben usw., obwohl diese nicht meinend erfasst ist. Dieses das, was der Positivismus als reine Gegebenheit meint. Aber ein Produkt der Abstraktion. Auch hier das Problem von II- nach den Seinssphären und den Arten des Gegebenseins ».

17. Notre interprétation s'appuie ici sur le manuscrit de 1907, dans lequel Daubert accuse notamment Natorp de réduire les couleurs essentiellement à des oscillations d'ondes, négligeant ainsi leur représentation et leur statut d'objet dans la perception. Concernant ce manuscrit, dans lequel sont regroupés les manuscrits A I 1/32, 3 et 31, voir nos explications dans Fréchette, 2000, pp. 93-110.

18. À l'appui, Daubert revient sur la question des pures donations positivistes dans un texte de 1930 (Ms. A I 3/30v), dans le contexte où la phénoménologie transcendantale husserlienne doit rendre compte de la donation dans le régime de la constitution : «Wenn letzteres der Fall wäre, so müßte die eigentliche Selbstgegebenheit abstrahiert oder rekonstruiert werden nach Art der positivistischen reinen Gegebenheiten ". Autrement dit, dans la mesure où la conscience entre dans le domaine réservé auparavant à la donation, l'essence et la vision des essences telle qu'elle était comprise dans les Recherches logiques sont réduites à des évidences de types positivistes.

19. "Dies also suchen Avenarius, Mach und Husserl gemeinsam: eine neue Unmittelbarkeit zu den Sachen selbst; eine neue Sachtreue in der Theorie ; eine neues Verständnis des subjektiven Weltbezugs; eine neue Art von Evidenz ». Sommer, 1985, p. 13. Dans Sommer, 1987, on trouvera une étude approfondie de ce qui pourrait s'avérer être une phénoménologie " positiviste". 
Cette similitude dans la recherche ne se prolonge toutefois pas jusqu'aux principes qui la sous-tendent, notamment en ce qui a trait à l'apriorité de la nature, le concept naturel de monde ainsi que les sciences de la nature, qui sont des domaines pour lesquels le positivisme, celui de Avenarius à tout le moins, jure avec la phénoménologie. Dans un texte de 1910/ $11^{20}$, où il tente justement de distinguer les nouveaux développements de sa phénoménologie par rapport au positivisme, Husserl critique la théorie de l'expérience pure d'Avenarius en tant qu'elle présuppose que le concept naturel de monde représente le monde tel qu'il existe dans l'effectivité, une présupposition qui ne serait pas celle de la phénoménologie :

Que l'idée de la nature puisse trouver à s'appliquer à la nature donnée, présuppose précisément une nature donnée; mais, si une nature est là effectivement, existe dans l'effectivité, c'est quelque chose de constamment tenu en réserve. L'apriorité veut dire : si nous partons du fait de l'attitude naturelle et du fait de la nature saisissable en elle et caractérisable de manière générale, et du fait que cette thèse a son droit non douteux, alors tout énoncé de la science de la nature est, en tant qu'énoncé qui détermine scientifiquement le particulier posé dans cette thèse, dépourvu de sens, s'il va à l'encontre du sens de cette thèse d'après sa teneur générale déterminante ${ }^{21}$.

Le positivisme d'Avenarius et de Mach se distingue également de la phénoménologie sur la question de l'évidence : la phénoménologie chercherait à identifier l'évidence à partir de la vie même, alors que l'évidence d'un point de vue positiviste est à comprendre comme la vie elle-même, et ne peut être inscrite dans un dualisme entre la conscience et le monde extérieur ${ }^{22}$.

20. Hua XIII/130-138.

21. Ibid., pp. 135-136. Traduction française tirée de Husserl, 1991.

22. Cette distinction nous est suggérée par Sommer, 1985, p. 19 : « Avenarius kennt nicht Evidenz im Leben, sondern nur Evidenz als Leben (...) Auch Mach kennt keine Evidenz im Leben, sondern nur Evidenz statt Leben : Sie ereignet sich im Augenblick der Selbstauflösung des Ich. Dies lernt Husserl in seiner Auseinandersetzung mit dem Positivismus : das Leben ist in sich selbst ». Il peut être pertinent de prolonger ce type de positivisme tel qu'entendu par Daubert jusqu'à celui suscité par le Tractatus de Wittgenstein : rappelons à cet effet la question de Kant à laquelle Dummett, dans ses conférences de Bologne, donne la réponse frégéenne : « si nous n'avons aucune représentation ni intuition d'un nombre, comment peut-il jamais nous être donné ? (Dummett, 1991, p. 14. Voir également Frege, 1969, p. 188). Pour Frege, c'est en comprenant le sens des énoncés qui contiennent des termes de nombres que des nombres nous sont donnés (voir Dummett, 1998, pp. 64-65). Dans ce cas, la donation se fait par compréhension du sens d'un énoncé. Pour Wittgenstein, comprendre une proposition, c'est savoir ce qui arrive quand elle est vraie ; comme la proposition est une fonction de vérité des propositions élémentaires, on peut dire que les propositions élémentaires sont en contact direct avec la réalité. C'est de cette façon que se développe l'ontologie des faits atomiques, et les propositions élémentaires wittgensteiniennes, ainsi que les nombres frégéens, relèveraient ainsi de la pure donation telle que Daubert l'entend. D’un point de vue phénoménologique, en remontant la chaîne ontologique de Wittgenstein, les états de chose tels qu'ils sont compris dans le Tractatus découlent d'une déduction partant de l'espace logique et qu'ils sont en ce sens partie intégrante de cette donation pure. 
Ainsi, la pure donation telle que l'entend le positivisme est comprise comme un élément non distinct de la vie même, ce que pourront comprendre certains lecteurs du Tractatus de Wittgenstein dans la définition du monde comme l'ensemble des faits ${ }^{23}$.

Il y a cependant une distinction de méthode entre la phénoménologie et le positivisme, et c'est celle de l'abstraction, que le positivisme ne pratique pas dans la mesure où il refuse le dualisme conscience/monde extérieur. Or, la phénoménologie doit procéder parallèlement en deux méthodes : elle s'intéresse aux objets tels qu'ils sont isolés dans leur donation pure en tant $q u$ 'indépendants de toute forme de représentation ou d'intuition sensible ( "même si ceux-ci ne sont pas saisis en visant »). C'est là la détermination positiviste de la phénoménologie. Mais la phénoménologie procède également par abstraction, qui est la procédure inverse du positivisme en ce sens : elle consiste à pouvoir viser un même objet selon un mode de conscience nouveau, par lequel c'est précisément l'espèce, au lieu de l'individuel, qui devient objet pour nous ${ }^{24}$. Par ce parallélisme des méthodes, la phénoménologie dans sa détermination objective est confrontée au problème suivant : considérant que l'évidence est définie par l'adéquation entre le donné et le saisi et que ce qui est visé est également donné ( $2^{\text {ème }}$ détermination), une telle évidence en termes d'adéquation ne peut être d'aucune valeur pour la connaissance, sinon qu'en correspondant à l'état-de-chose de la nature donnée, ce que fait précisément l'évidence de type positiviste. Sur ce plan, la phénoménologie n'apporterait rien de plus que le positivisme ${ }^{25}$. La solution réside alors dans l'agencement des modes d'évidence et de la saisie.

La quatrième détermination de la phénoménologie est la suivante :

Comme doctrine des réalités de donation les plus immédiates. Espèces, relations, fondations, etc. doivent se construire, selon leur nature, sur quelque chose, sans quoi elles ne peuvent entrer en relation avec leur existence. Par ces fondements, on peut poser la question des conditions de leur existence et de

23. Sur la portion inverse de la relation que nous avons évoqué entre Wittgenstein et la phénoménologie, notons que dans ce qu'on pourrait appeler un tournant vérificationniste chez Wittgenstein (1929-30), celui-ci distingue trois sortes de propositions : les genuines propositions (Aussagen), les hypothèses et les propositions mathématiques. Or, les genuine propositions, ont sens (sont vérifiées ou falsifiées) en ce qu'elles sont comparées avec la réalité, ce qui correspond en fait aux propositions élémentaires du Tractatus. Elles correspondent selon Wittgenstein à un "langage phénoménologique ", qui plus est à une grammaire phénoménologique, qui investigue les règles linguistiques. Voir à cet effet Glock, 1996, pp. 383384, ainsi que Marion, M., 1998, sur la question du langage phénoménologique.

24. Dans les Recherches logiques, Husserl décrit le processus d'abstraction comme suit : "Quand nous visons le rouge in specie, un objet rouge nous apparaît, et c'est en ce sens que nous portons notre regard sur cet objet (que nous ne visons pas cependant). En même temps surgit en lui le moment rouge, et, dans cette mesure, nous pouvons dire ici également que nous portions notre regard sur lui ». Hua XIX/1/111.

25. Voir Fréchette, 2000a, sur les conséquences de cette position dans un cadre naturaliste. 
leur donné-même. On atteint ensuite un cercle défini de phénomènes, d'abord ceux de la sensibilité, mais également une unité définie, etc. catégoriale ${ }^{26}$.

Cette quatrième détermination est celle de l'ontologie. Nous pouvons penser que Daubert reprend en partie certaines des tâches que la phénoménologie des Recherches logiques s'était fixée, à savoir la constitution d'une ontologie, et plus spécifiquement, d'une théorie de l'objet. Comme nous l'avons vu, la troisième détermination renvoyait à l'abstraction, qui consiste à passer de l'individu à l'espèce en distinguant puis isolant l'identique dans l'objet visé, puis en le visant non plus comme identique de l'objet visé mais comme identique comme tel. Si cette troisième détermination renvoie plutôt explicitement à la seconde des Recherches logiques, la quatrième détermination, celle des différences entre objets, semble se rapprocher davantage des préoccupations de la troisième Recherche logique : qu'il s'agisse d'espèce, de relation, elles renvoient toutes directement à la théorie de l'objet de la troisième Recherche logique ${ }^{27}$ : cette quatrième détermination est donc celle de la théorie de l'objet, des donations dans leur réalité et de leur fondation, ce qui concerne les concepts tels que ceux d'espèce, de relation, etc. qui doivent être construits sur quelque chose selon leur nature, autrement dit en tant qu'objets. Ici, il semble que Daubert tente de s'écarter d'un réalisme direct tel qu'il est pratiqué par exemple par Reinach : la condition de relation entre la réalité de donation et l'existence passe par une théorie de l'objet, ainsi que par une mise au clair des concepts qui sont garants des catégories de l'objet. Une fois cette condition respectée - celle de la détermination des concepts de tout et de partie $(\$ 21$, IIIe $R l)$ - celle de l'existence et du donné-même de l'objet peut être posée, cette deuxième condition étant celle des formes d'unité sensibles et catégoriales ( $\mathbb{\$} 22-23$, IIIe RL). Ce processus permet de passer des donations aux objets dans les donations ainsi qu'aux phénomènes par lesquels ils sont donnés, ce que Daubert conçoit comme un domaine catégorial.

La cinquième détermination concerne la phénoménologie comme doctrine de la structure de donation au sens de la relation de fondation. Le concept de fondation est défini par Husserl au chapitre II de la troisième

26. «Als die Lehre von den nächsten Gegebenheitsrealitäten. Spezies, Beziehungen, Fundierungen usw. müssen sich ihrer Natur nach auf etwas aufbauen, ohne das sie nicht in ihr Dasein treten können. Bei diesen Fundamenten kann man nach den Bedingungen ihres Daseins und ihrer Selbstgegebenheit fragen. Dann landet man bei einem bestimmten Kreis von Phänomenen, zunächst denen der Sinnlichkeit, dann aber auch bestimmter kategorialer (Einheit usw.) ».

27. En introduction à sa troisième Recherche logique, Husserl affirme : « Il faudrait donc placer systématiquement sa discussion [celle de la différence entre contenus abstraits et contenus concrets] dans la théorie pure (a priori) des objets comme tels, dans laquelle on traitera des idées ressortissant à la catégorie de l'objet, comme tout et partie, sujet et propriété, individu et espèce, relation et collection, unité, quantité, série, nombre ordinal, grandeur, etc., ainsi que des vérités a priori qui se raportent à ces idées " (Hua XIX1/227). 
Recherche logique, où il présente une théorie des formes pures en six théorèmes basés sur la définition suivante :

$\mathrm{Si}$, conformément à une loi d'essence, un a ne peut exister comme tel que dans une unité qui l'embrasse et qui le relie avec un $\mathrm{m}$, nous disons qu'un a comme tel a besoin d'être complété par un $\mathrm{m}$. Si, en conséquence, $\mathrm{a}_{\circ} \mathrm{m}_{\mathrm{o}}$ sont des cas singuliers déterminés, réalisés dans un seul tout, des genres purs a et $\mathrm{m}$ se trouvant dans le rapport indiqué, nous dirons que $a_{\circ}$ est fondé par $m_{\circ}$ et exclusivement fondé par $m_{\circ}$ quand seul $m_{\circ}$ peut satisfaire ce besoin d'a d'être complété. De manière moins déterminée, nous dirons, en outre, que les deux contenus ou les deux espèces pures ont entre eux un rapport de fondation (Fundierungsverhältnis), ou encore de connexion nécessaire ${ }^{28}$.

Ainsi, la relation de fondation telle que l'entend Husserl se définit par l'impossibilité, en regard d'une loi a priori, de penser l'existence d'un individu sans celle d'un second qui fonde l'existence du premier. Or, pour Daubert, la cinquième détermination de la phénoménologie s'énonce comme suit :

(...) la phénoménologie comme doctrine de la structure de donation au sens de la relation de fondation selon sa proximité et sa distance et selon les relations de fondation et de dépendance s'y fondant. En plus, les sphères d'être au sens des sphères de donation. Ici, les différences d'abstraction et d'idéation. Cela ne se recouvre pas totalement avec II- et III- ${ }^{29}$.

La doctrine de la structure de la donation est comprise ici comme théorie de la forme telle qu'elle est présentée chez Husserl, et en ce sens, la donation est à comprendre comme une relation de fondation. La phénoménologie est en ce sens une théorie des formes de donation, et Daubert semble ici insister surtout sur le niveau des espèces dans la structure de la donation, en mentionnant les concepts d'abstraction et d'idéation ${ }^{30}$ : on peut dire de ces concepts qu'ils sont corrélatifs dans la mesure où l'abstraction est à comprendre comme le processus donnant l'espèce, l'idéation étant le processus par lequel un acte vise l'espèce comme telle. Il s'agit effectivement de deux actes différents, et au demeurant liés par une relation de fondation :

28. Hua XIX1/261. Traduction française tirée de Husserl, 1963.

29. «(...) Die Phänomenologie als Lehre von der Gegebenheitsstruktur im Sinne der Fundierungsbeziehung nach ihrer Nähe und Ferne und den hierin gründenden Abhängigkeitsund Fundierungsbeziehungen. Damit Seinssphären im Sinne von Gegebenheitssphären. Hier die Unterschiede von Abstraktion und Ideation. Es deckt sich dieses wohl nicht mit II- und III- ».

30. Il n'est pas clair que Husserl, dans les Recherches logiques à tout le moins, distingue vraiment l'abstraction de l'idéation. Le terme d'abstraction est utilisé pour décrire le processus subjectif vers les actes, alors que l'idéation est le terme utilisé pour décrire les actes résulant du processus d'abstraction. Voir à cet effet tout particulièrement Hua XVIII/109, 135 ; XIX/1/108, 149 ; XIX/2/733, 765. Dans les Ideen, cette distinction est présentée autrement et Husserl décrit l'idéation comme vision d'essence (HuaIII/13). Plus loin, il commente : "Dans les Recherches logiques, j'ai employé d'ordinaire le mot idéation pour désigner l'intuition eidétique qui donne de façon originaire et même de préférence pour désigner sa forme adéquate. 
l'idéation est dépendante de l'abstraction, et cette relation de dépendance délimite différents domaines ou sphères de donation qui sont l'objet de la phénoménologie en tant que théorie des formes. Si cette détermination ne recouvre pas entièrement les II- et III-, c'est que dans sa détermination en tant que théorie des formes, la phénoménologie considère la structure de la donation comme telle indépendamment des donations individuelles, d'où l'importance de l'abstraction et l'idéation.

La sixième détermination de la phénoménologie est à comprendre comme :

doctrine des relations d'essence : 1 ) des essences et de leurs saisies, pour autant qu'elles peuvent valoir comme point de relation pour les relations visibles. 2) La nature de cette relation dans de mêmes sphères et dans des sphères différentes (relations de fondation). 3) Les donations et les saisies de modes différents, qui sont conditionnées à ces relations, en tant que visions de nature différente. Une extraction particulière du formel-catégorial propre ${ }^{31}$.

Ici, Daubert n'insiste plus tant sur la donation que sur l'essence telle quelle et ses relations : premièrement les saisies en tant qu'elles sont des relations d'essences ; deuxièmement, la nature des relations, ce qui a été présenté plus haut par la théorie des formes; enfin, un type particulier de donation, ce qui est étudié dans la sixième Recherche logique, l'intuition catégoriale. Ces trois points concernant la sixième détermination insistent à nouveau sur la question d'intuitions ou de visions de nature différentes, comme c'était le cas dans la première détermination. Aussi, ce n'est pas par la supposition d'une autre forme de conscience que ces intuitions seront prises en compte, mais par le développement de la phénoménologie comme doctrine des relations d'essence.

Le point sept et la conclusion de ce manuscrit tentent davantage de mettre en ordre ce qui a été présenté auparavant que de donner d'autres déterminations de la phénoménologie. Aussi, Daubert affirme :

Parcourant tout ce qui a été vu jusqu'à présent, la question de ce qu'on appelle l'essence : non pas simplement II- et IV-, mais aussi les idéalisations et les

Cependant nous avons manifestement besoin d'un concept plus souple qui englobe toute conscience dirigée simplement et directement sur une essence pour la saisir et la poser, y compris également toute conscience obscure, dépourvue par conséquent de toute portée intuitive " (III/ 15). En fin de première partie, il poursuit : «(...) dans l'acte spontané d'abstraire, ce n'est pas l'essence mais la conscience de l'essence qui est un produit de l'esprit ; la situation est alors la suivante : il est manifeste au point de vue eidétique que la conscience donatrice originaire d'une essence (autrement dit l'idéation) est en elle-même et nécessairement spontanée (....) » (III/50). Traduction française tirée de Husserl, 1950.

31. «Phänomenologie als Lehre von den Wesensbeziehungen : 1. Von den Wesen und ihren Fassungen, soweit sie als Beziehungspunkt für einsichtige Beziehungen gelten können. 2. Die Natur dieser Beziehung in gleichen Sphären und in verschiedenen Sphären (Fundierungsbeziehungen). 3. Die damit bedingten verschiedenartigen Gegebenheiten und Erfasstheiten als Einsichtigkeiten verschiedener Natur. Besondere Herausschälung des eigentlichen kategorialen Formalen ». 
empreintes comme telles ont leur essence, et non seulement ce sur quoi elles sont construites. Une question particulière, à savoir si l'impropre a aussi son essence ou non (vraisemblablement non). À quoi s'oppose l'essence (c'est la question cardinale de la phénoménologie) ? 1. Comme soi en face d'un non soi. 2. Comme immédiat en face d'un fondé. 3. Comme pour soi, hors de toute fonction, sub specie aeterni, en face de sa fonction. 4. Comme le visible en face du hasard de la manifestation empirique (cette dernière opposition ne doit pas être comprise comme celle entre l'irrationnel et le rationnel. Précisément, c'est une illusion néfaste que de croire que les visions des domaines les plus différents sont réductibles au domaine rationnel) $)^{32}$.

\section{Et Daubert conclut :}

La phénoménologie comme doctrine des essences, c'est-à-dire doctrine de ce qui est visible, doit supplanter le rationalisme ou plus exactement le restreindre à son domaine formel, qui n'est qu'un cas particulier de la phénoménologie. Plus encore, la doctrine des essences comme doctrine des relations d'essence n'est qu'une partie de la vision générale, qu'elle soit des essentialités isolées ou liées les unes aux autres. Husserl, lequel venait du rationalisme, s'y limitait et considérait lui-même cela comme quelque chose de rationaliste ${ }^{33}$.

L'observation de ces six déterminations de la phénoménologie amène Daubert à conclure que la question litigieuse demeure dans tous les cas ce qu'on appelle l'essence en phénoménologie. Aussi, l'essence n'est pas seulement ce qui est donné, et ce n'est pas seulement les formes de donations. Les idéalisations et les empreintes ont également leur essence, ce qui, comme nous le verrons dans l'étude du troisième manuscrit, est une des conditions permettant à la phénoménologie de supplanter le rationalisme. Sur un autre plan, la définition de l'essence est problématique. L'essence se place en opposition à différents modes, et prend à son tour différents modes, ce qui ne nous permet pas de donner une définition claire et précise de l'essence. Au contraire, l'essence est toujours à l'opposé de l'objet étudié et recherché par la phénoménologie, et en ce sens l'essence ne peut être la chose elle-même. C'est en ce sens que

32. «Durch alles Bisherige hindurchgehend die Frage nach dem, was "Wesen ” heißt : Nicht einfach II- und IV-. Auch Idealisationen und Gepräge als solche haben ihr Wesen und nicht nur das, worauf sie gebaut sind. Besondere Frage, ob auch das Uneigentliche sein Wesen hat oder nicht (wahrscheinlich nicht). Wozu steht " Wesen ” im Gegensatz (dieses die kardinale Frage der Phänomenologie) ? 1. Als selbst gegenüber nicht selbst. 2. Als nächstes gegenüber Fundierten. 3. Als für sich, außer Funktion, sub specie aeterni, gegenüber seiner Funktion. 4. Als das Einsichtige gegenüber dem Zufall des empirischen Auftretens (dieses letztere darf nicht als irrational gegenüber rational gefaßt werden. Es ist gerade ein verhängnisvoller Irrtum, daß man die Einsichtigkeiten der verschiedensten Gebiete auf rationale zurückführbar glaubte) ».

33. «Phänomenologie als Wesenslehre, d.h. Lehre von dem, was einsichtig ist, muß den Rationalismus verdrängen oder richtiger einschränken auf sein formales Gebiet, das nur ein Sonderfall der Phänomenologie ist. Ferner die Wesenslehre als Lehre von Wesensbeziehungen ist nur ein Teil von überhaupt einsichtigen, sei es isolierten Wesenheiten oder zueinander bezogenen. Husserl, welcher vom Rationalismus kam, beschränkte sich hierauf und sah dieses selber auch für rationalistisch an ». 
Daubert présente la question de l'opposition de l'essence comme la question cardinale de la phénoménologie : la première opposition est celle de la propriété : l'essence est ce qui est propre (eigentlich) contre l'impropre. Elle est également ce qui est immédiat, en opposition à ce qui est fondé ; elle est pour elle-même, hors fonction, contre sa fonction. Enfin, elle est ce qui est visible, et cette vision ne la réduit pas à du rationnel, mais au contraire doit être considérée au sens le plus large, le rationnel étant un des domaines du visible. Ainsi, Daubert conclut en affirmant que la phénoménologie est une doctrine de ce qui est aperçu, et qu'elle dépasse par ce fait le rationalisme. Mais les relations d'essence, qui intéressent la phénoménologie dans ce qui a trait à sa théorie des formes, ne sont qu'un aspect de la vision, et la phénoménologie husserlienne, selon Daubert, ne serait pas à la hauteur même de son principe, car elle réduit le domaine des essentialités au rationnel, qui doit être conçu comme une partie de la phénoménologie, mais non pas comme sa fondation.

\section{La phénoménologie et les déterminations de l'évidence}

Cette critique du « rationalisme » de la phénoménologie husserlienne apparaît dans plusieurs manuscrits de Daubert, dont ceux que nous abordons maintenant, nommément celui intitulé phénoménologie et évidence (Phänomenologie und Evidenz) (A I 1/22), ainsi que le phénomène comme donné-même (Das Phänomen als Selbstgegebenes) (A I 1/32-31). Nous avons insisté, dans l'étude du manuscrit précédent, sur le fait que Daubert y conçoit la phénoménologie comme une doctrine des essences, bien que le concept d'essence développé par Daubert soit plus large que celui de Husserl. Nous avons pu également voir, dans le manuscrit précédent, que se trame un double régime dans ce que Daubert conçoit comme la donation : d'une part, le donné tel qu'il est l'objet de la saisie, ce que Daubert entend par "phénomène au sens de la saisie "; d'autre part, le donné comme phénomène, indépendamment de sa saisie, ou encore la teneur ou qualité de donation. En A I 1/22, c'est dans l'adéquation du donné et du saisi que le phénomène pourra être dit évident :

I. Le phénomène est le donné pur, la teneur de donation (ce que veut donner Husserl par la réduction phénoménologique). II. Le phénomène est la saisie, dans laquelle ce donné-là m'apparaît. Dans cette saisie se fondent des relations d'essences de différents types. III. Le phénomène est le donné, pour autant qu'il est adéquat à une saisie déterminée (pour autant que le pensé ou le saisi soit donné en même) $)^{34}$.

34. «I. Phänomen ist das rein Gegebene, der Gegebenheitsgehalt (das, was Husserl durch phänomenologische Reduktion geben will). II. Phänomen ist die Fassung, in welcher jenes Gegebene mir erscheint. In dieser Fassung gründen Wesenszusammenhänge verschiedener Art. III. Phänomen ist das Gegebene, sofern es einer bestimmten Fassung adäquat ist (sofern das Gemeinte oder Erfaßte auch gegeben ist) ». 
Cette adéquation du donné et du saisi est un point central de la phénoménologie, et Daubert renvoie directement à ce que Husserl « veut donner » par la réduction phénoménologique : comme nous l'avons exposé en introduction, la réduction vise d'une part à rendre la conscience originaire et " absolument donnée » ${ }^{35}$, et d'autre part à rendre l'objet du phénomène dans sa donnée pleine ${ }^{36}$, qui est donnée par la réduction non seulement comme corrélat des modes d'apparition subjective possibles mais comme "constituable » dans l'identité noématique, ce que Daubert exprime dans l'extrait cité ici comme la "teneur de donation» (Gegebenheitsgehalt). En d'autres termes, il s'agit ici du donné tel quel, indépendamment de la saisie, qui peut m'être donné de différentes manières, correspondant à différents degrés d'évidence ${ }^{37}$, mais qui n'implique dans aucun cas, selon Daubert, une réduction qui puisse établir un point de vue privilégié sur les objets, et les donnant ainsi dans une plus grande "pureté ». Au contraire, s'il y a une teneur déterminée des saisies, ce qui lui correspond ne donne pas accès, à travers la réduction phénoménologique, à un type d'évidence supérieur à l'adéquation, mais « à une théorie des relations d'essence au sens de l'a priori, sensible comme catégorial ${ }^{38}$. Aussi, que le phénomène soit un donné, cela n'en tient qu'à l'adéquation de celui-ci avec une saisie déterminée. Cependant, comme Daubert le remarque : "Ici la question demeure malgré tout encore ouverte, à savoir qu'appelle-t-on phénoménologie et donné-même, c'est-à-dire qu'est-ce que je dois regarder pour apercevoir quelque chose ? ". C'est ici que Daubert retient son attention sur le concept de saisie, qui comme tel peut contenir certaines présuppositions : " la saisie ressemble encore à l'apperception et à la position active et arbitraire de la raison. Ce psychologisme ou activisme doit tomber ${ }^{39}$. Ces deux dernières citations soulèvent deux questions : d'une part, celle du critère d'objectivité pour le

35. Voir entre autres HuaIII1/96-99.

36. Cette expression (Gegebenheitsfülle) renvoie aux différents modes de donation et aux différences qui se trouvent dans la sphère de la perception (HuaIII1/94), et qui correspondent aux différents degrés de clarté (Klarheitsgrade, Abschattungsweise) du phénomène, et donc de la qualité de son évidence.

37. Cette explication est fournie par Daubert dans une remarque en marge du passage cité : « Dem entsprechen ganz verschiedene Evidenzarten, entsprechend dem, was evident ist, da dieses ja in ganz verschiedener Weise für mich da ist».

38. Nous citons ici la majeure partie du paragraphe auquel appartient cette phrase : «Im Sinne von III. gibt es hinsichtlich eines bestimmten Gehalts der Fassungen eine Lehre von Wesenszusammenhängen im Sinne des Apriori, sinnlichen wie kategorialen. Diese gründet sich immer auf einen zu vermeidenden Widerspruch in den Sachverhalten und setzt voraus jene begriffliche Fassung (das Gepräge hinsichtlich der Gegenstandswelt), für welche streng der Satz der Identität und des Widerspruchs gilt. Offen ist hierbei allerdings noch die Frage, was hier Phänomenologie und Selbstgegebenheit heißt, d.h. worauf ich eigentlich hinblicken muß, um etwas einzusehen".

39. " Fassung" sieht noch etwas nach Apperzeption und aktiver willkürlicher Vernunftsetzung aus. Dieser Psychologismus oder Aktivismus muß wegfallen ». 
donné-même, ce qui fait intervenir nécessairement des objets privilégiés et, d'autre part, comment peut-on distinguer, à partir du donné, dans le donnémême pour ainsi dire, ce qui est saisi, et de quelle manière ? Cette première question implique la seconde, en ce que la raison, ou un ensemble de normes préétablies, doit être supposé afin de rendre ces distinctions compréhensibles. Cette citation renvoie manifestement à un passage du manuscrit étudié précédemment, à savoir l'accusation de rationalisme prononcée par Daubert contre la phénoménologie de Husserl. Ici, non seulement cette accusation est indirectement réitérée, mais Daubert l'achève en identifiant la forme de rationalisme pratiquée par Husserl en phénoménologie à un psychologisme ou un activisme, ce qui rabat la phénoménologie transcendantale sur les premières thèses de Husserl dans la Philosophie de l'arithmétique. Aussi, il juge important de distinguer, à l'intérieur de la saisie et de la position, le caractère arbitrairement applicable de la fonction de connaissance, contre ce qu'elle pourrait avoir, d'un point de vue activiste, d'arbitraire en $\operatorname{soi}^{40}$.

Ce problème de la saisie du donné et des paramètres, pourrait-on dire, de la position (Setzung) et de ce qu'on doit regarder pour apercevoir quelque chose, comme l'écrivait plus haut Daubert, est alors la question centrale de l'évidence en phénoménologie : en ce qu'elle est une forme d'adéquation entre le donné et le saisi, elle est réglée selon les conditions respectives de l'apercevabilité (Einsichtigkeit). Et il y a ici un choix à faire, nommément entre les conditions de l'apercevabilité, en d'autres termes les relations d'essence, et le matériel même des saisies, qui dans la mesure où la première option est choisie, demeure en quelque sorte hypothétique ${ }^{41}$.

Daubert commente ensuite le second problème et revient sur celui de la réduction phénoménologique, tel qu'ils étaient énoncés en introduction du manuscrit, nommément le phénomène compris comme saisie (Fassung), dans laquelle un donné m'apparaît. Dans ce contexte, je peux me rendre clair l'objet, pour autant qu'il est saisi ou qu'il m'apparaît, bien que cela soit encore indépendant de la question, c'est-à-dire si l'objet pensé est véritablement ainsi ${ }^{42}$. Dans cette mesure, l'évidence et la clarification sont complè-

40. «Es bleibt etwas an der "Setzung " und "Fassung” als etwas Aktives daran. Die Aktivität ist hier die Erkenntnisfunktion, die allerdings nichts willkürlich für sich, sondern nur willkürlich anwendbar ist ».

41. «Evidenz bezieht sich hier auf die Einsichtigkeit dieser Fassungen oder Gepräge, aber nur hinsichtlich ihrer Beziehungen zueinander. Dabei besteht das Problem, daß das jeweilige Material dieser Fassungen für die Einsichtigkeit ihrer Wesensbeziehungen eigentlich gar nicht mehr in Betracht kommt, es ist gleichsam hypothetisch. Damit hat es eine ganz besondere näher zu analysierende Rolle ».

42. "Diese meine Meinung oder der Gegenstand, sofern er erfaßt ist oder mir erscheint, kann ich mir klarmachen. Aber dieses ist noch unabhängig von der Frage, ob der gemeinte Gegenstand wirklich so ist. Ganz anders ist die Klärung und die Evidenz, wenn ich frage, ob das Gemeinte, welches sich mir in der Erscheinung darstelle, wirklich so ist. Dann suche ich es mir zur Selbstgegebenheit zu bringen ». 
tement différentes de ce qu'elles seraient lorsque l'identité de l'objet n'est pas mise en cause.

Le problème de la réduction phénoménologique, en ce qui a trait à l'évidence, avait déjà été thématisé plus haut dans le manuscrit, et Daubert revient ici sur la position sous-entendue par l'accusation portée contre la réduction : le donné n'est dans les faits jamais isolé ou donné de façon autonome pour soi, mais au contraire toujours comme matériel d'une saisie : il doit être extrait (herausgeschält) de cette saisie ${ }^{43}$. Reste à savoir en quel sens le donné doit être extrait. Par extraction, Herausschälung, Daubert n'entend pas la solution husserlienne de la réduction de l'objectivité telle que présentée dans les Ideen..., mais bien l'extraction à même les différents modes de donation, dont celui du "formel catégorial propre ", telle qu'elle était entendue par la sixième détermination de la phénoménologie dans Les déterminations possibles de la phénoménologie. D'autre part, la saisie ne contient pas le matériel tel qu'il est donné, jusque dans ses nuances sensibles, et en ce sens, "partant de la saisie, je ne peux donc pas réduire ${ }^{44}$.

Ces deux points concernant le problème de la réduction phénoménologique présentent les tâches phénoménologiques possibles à appliquer au donné : d'une part l'extraction, d'autre part la réduction. D'une part, l'extraction implique que le donné est toujours matériel d'une saisie, bien que la saisie ne contienne pas nécessairement, ou absolument, le matériel tel qu'il est donné. C'est en ce sens, d'autre part, que la réduction peut constituer une seconde possibilité, mais d'emblée déjà diminuée en ce que sa tentative se base sur une forme d'optimisme injustifié, à savoir celui d'un objet donné indépendamment de toute saisie, ou en termes néokantiens, d'un objet en soi, d'un $x$.

Enfin, dans la troisième partie du manuscrit, Daubert met en parallèle les questions précédentes avec celle de la donation des choses, des objets et des relations, ainsi que la place fonctionnelle de la chose et de l'objet dans la fonction de connaissance ou de donation. Cette question implique la fonction des saisies, que Daubert nomme ici la concrétion, qui est plus précisément un moment de la fonction de connaissance des saisies. Ici, c'est le problème de la fonction de connaissance de la saisie qui est mis en jeu, et Daubert se demande comment est-il possible de dire dans le même sens d'un côté que des choses, des objets sont donnés, et de l'autre que des relations factuelles le sont. Vraisemblablement, c'est qu'il faut séparer la question de l'évidence de celle de la place des choses et des objets dans la fonction de con-

43. "Tatsächlich ist das im letzten eigentlichen Sinne Gegebene nie isoliert oder selbständig für sich gegeben, sondern immer als Material einer Fassung. Es muß also dieser Fassung herausgeschält werden ».

44. Cette phrase apparaît à l'intérieur de ce paragraphe : «Andererseits aber enthält diese Fassung nicht genau das Material, so wie es gegeben ist (z.B. statt sinnliche Nuance ein allgemeines Weiß). Aus der Fassung heraus kann ich also nicht reduzieren ». 
naissance ou de donation ${ }^{45}$. Cette séparation implique une distinction de différents concepts d'évidence et de donation, que Daubert tente d'éclaircir : d'une part, le donné-même, au sens le plus large, peut être le quid au sens de n'importe quelle unité, mais uniquement les relations et les relations d'étatde-chose peuvent être dites évidentes en ce sens ${ }^{46}$. Cette distinction est majeure, en ce qu'elle donne d'une part au concept d'évidence une affectation à certains niveaux ontologiques : autrement dit, elle ne peut pas s'appliquer à toutes sortes d'objets, bien que toutes sortes d'objets soient sujets à être donnés, et à être considérés comme du donné-même. Aussi, en ce sens, l'évidence semble pouvoir s'appliquer essentiellement au niveau catégorial. D'autre part, cette distinction n'évacue pas la possibilité d'une évidence factuelle, mais celle-ci, se fondant sur un donné-même, ne peut pas concorder avec un donné-même simple ${ }^{47}$. Bien qu'en différenciant deux types d'évidences, Daubert ne procède à aucune réduction, mais à ce qu'il nomme l'extraction, qui n'est pas sans faire penser à la première théorie de l'abstraction dans les $R l$ de Husserl.

Daubert reprend en fin de manuscrit les définitions de départ du terme de phénomène, à propos de ce qu'il appelle le double sens du phénomène ou de l'apparition (Doppelsinn von Phänomen oder Erscheinung) : le premier sens représente ce qui est saisissable en même, dans une pure forme de donation, analysé selon ce qui lui est constitutif. C'est la théorie des objets déterminés selon ce qu'ils sont en eux-mêmes. Ils deviennent ensemble des phénomènes par le "même ". Différentes sphères d'être lui appartiennent par le «donné ", en face de quoi se tient le saisi. Le second sens représente ce qui est en tant que phénomène pour moi, pour autant qu'il est donné actuellement, ce que Husserl, selon Daubert nomme l'objet phénoménal, pour autant qu'il soit un phénomène ${ }^{48}$.

45. " Es schließen sich an die Fragen, wie einerseits Dinge und andererseits Gegenstände und andererseits sachliche Relationen gegeben sind. Ferner hiermit zusammen die funktionale Stellung von Gegenstand und Ding in der Erkenntnis- (Gegebenheits-) funktion. Davon zu trennen ist die eigentliche Frage der Evidenz ».

46. «Selbstgegeben im weitesten Sinne kann bestimmtes Was im Sinne irgendwelcher Einheit sein. Evident aber können nur Beziehungen und zwar Sachverhaltsbeziehungen sein ».

47. « Hier nun der ganze Unterschied zwischen kategorialer Evidenz im Sinne der zu verstehenden gegenstandstheoretischen Prinzipien (logische Gesetze) und tatsächlicher Evidenz. Letztere als Konstatierungsevidenz. Diese mag sich auf Selbstgegebenes gründen, aber fällt nicht zusammen mit einem einfach Selbstgegeben ».

48. "Doppelsinn von Phänomen oder Erscheinung : 1. Dasjenige, was überhaupt in reiner Gegebenheitsform (selbst) faßbar ist, analysiert nach dem, was ihm konstitutiv ist. (Dabei fällt also die Frage, ob das Wirkliche immer erfaßt ist oder nicht, Tonhöhe und Farbe usw. fort.) Lehre von bestimmten Gegenständen nach dem, was sie selbst sind. Gemeinsam zu Phänomenen werden sie durch das " Selbst". Verschiedene Seinssphären gehören in es durch das "Gegeben ”. Dem steht immer gegenüber das Erfaßt. 2. Dasjenige, was jetzt gegeben ist, in seiner Modalität oder als Erscheinung für mich (Husserls phänomenaler Gegenstand, sofern es Phänomen ist). » 
Enfin, nous aimerions ici porter notre attention sur d'autres extraits des manuscrits de la Mappe 1, à savoir le A I 1/32-3149, qui développent également cette question du donné en phénoménologie. À la fin du A I 1/ 32 (qui précède le A I 1/31 dans la numérotation des fascicules), Daubert pose la question kantienne, qu'il avait déjà posée dans le manuscrit sur les déterminations possibles de la phénoménologie : "comment la connaissance de la nature est-elle possible ? ». Il pose cette question dans le contexte d'une définition du phénomène comme "apparition d'un " en-soi " d'un objet de la nature ", servant dans la théorie de la connaissance précisément cette question kantienne ${ }^{50}$. Cette question kantienne se poursuit chez Natorp et l'école néokantienne, à laquelle Daubert oppose la phénoménologie en ce qu'elle est une "représentation de la nature telle qu'elle est pure pour nous (dans nos perceptions et dans notre relation tout à fait naturelle, sensible et compréhensive à la nature vivante qui nous environne) ", nature qui s'étend également aux objets sociaux, idéaux, historiques et culturels ${ }^{51}$. Aussi, la phénoménologie est ici comprise, en opposition au kantisme, comme une théorie de la nature telle qu'elle nous est donnée. Dans ce contexte, la phénoménologie doit être comprise comme une théorie du donné-même, et doit par le fait même exclure toute prédétermination du donné dans la saisie ("saisir quelque chose tel qu'il est en soi »), ce qui reviendrait à adopter la position de Kant et de Platon sur la présence de l'idée déjà dans la vision ${ }^{52}$.

Contre cette issue platonicienne ou kantienne, Daubert propose l'alternative suivante : certes, les phénomènes ne sont certainement pas totalement indéterminés, et sont toujours d'une certaine manière constituables. Seulement, certaines marques ou formes sont incarnées dans les donations, elles leur sont naturelles, et cela construit le régulatif naturel pour toutes les mesures scientifiques et pratiques ainsi que pour les constitutions qui s'y lient. L'erreur consiste à confondre les déterminations qui se trouvent dans les formes naturelles qui nous sont données, avec les formes qui

49. Selon les indications de Daubert contenues à même le texte, il est justifié de penser que ce manuscrit fut rédigé entre 1908/09 et 1913.

50. Ce passage du manuscrit se présente ainsi : "Phänomen als Erscheinung eines “ Ansich " eines Naturgegenstandes diente in der Erkenntnistheorie gewöhnlich den kantischen Fragen : Wie ist Naturerkenntnis möglich ?".

51. "Auch Naturgegenständen gegenüber gibt es eine Phänomenologie als eine Darstellung der Natur so wie sie für uns rein ist (in unseren Wahrnehmungen und in unserem ganzen natürlichen sinnlichen und verstehenden Verhältnis zur lebendigen uns umgebenden Natur) ".

52. Daubert présente le problème ainsi : «Etwas zu fassen, so wie es an sich und selbst ist, scheint unmöglich zu sein, denn : 1. Wie man es auch fassen mag, es muß doch in der Fassung irgendwie bestimmt sein. [...] (Hier allgemeines und tiefstes Problem Kants und Platos. In der Anschauung schon die Idee) ». 
sont composées à travers les constitutions au sens d'un intérêt déterminé s3 $^{53}$. Cette distinction entre les formes telles qu'elles se trouvent dans les donnés, et les formes telles qu'elles sont constituées à partir des donnés, en insistant sur le premier type de formes, n'est pas sans faire penser aux qualités de forme (Gestaltqualitäten) telles qu'elles ont été développées par Ehrenfels ${ }^{54}$. Cette distinction était déjà présente dans le premier manuscrit que nous avons abordé, précisément sur la question de l'essence en regard des idéalisations et des empreintes : celles-ci ne sont alors pas à chercher dans des déterminations construites mais dans les déterminations et les formes incarnées à même les donations. Cette alternative permettrait alors à la phénoménologie de faire l'économie du rationalisme. Aussi, l'erreur du postkantisme (tel que Daubert l'entend) - et d'un certain type de phénoménologie — est de comprendre le monde comme mécanique rationnelle, et de rendre équivalent le donné naturel avec le matériel de la vision et la construction/constitution conceptuelle.

\section{4. Éléments de conclusion}

L'intérêt de Daubert pour la phénoménologie husserlienne s'est toujours tenu aux Recherches logiques, bien que celui-ci ait étudié attentivement les Ideen... ainsi que les travaux ultérieurs de Husserl. Comme on a pu le voir à l'étude de ces manuscrits, ce que nous avions annoncé en introduction comme le motif central de la phénoménologie, le fait de la donation des objets, se présente chez Daubert sur un arrière-fond sensiblement plus complexe que ce que l'introduction et le choix de la réduction phénoménologique dans les Ideen... de Husserl laisse entendre.

Rappelons d'abord les idées principales de ces manuscrits : en premier lieu, les donations sont subjectives, et nécessitent certes un regard différent sur les objets qu'elles contiennent, mais cette différence de regard n'implique en aucune façon d'appliquer une réduction tel que le fera Husserl. C’est ici que Daubert présente l'abstraction comme un principe dont la phénoménologie n'a peut-être pas épuisé toutes les ressources, du moins telles qu'elles étaient exposées dans les Recherches logiques. Aussi, comme Daubert le

53. Ce passage s’énonce comme suit : «Die Phänomene sind gewiß nicht einfach formlos unbestimmt, und sie sind für uns immer in gewissen Auffassungen da. Aber das Entscheidende ist dieses : Gewisse Gestaltungen oder Gepräge sind in den Gegenbenheiten inkarniert, sind ihnen natürlich, und diese bilden das natürliche Regulativ für alle wissenchafltichen und praktischen Messungen und Auffassungen, die sich darauf beziehen. Der große Fehler ist, zwei Arten von Auffassungen verwirrt zu haben oder besser : die Bestimmtheiten, die in den natürlichen Gestaltungen liegen, vermischt zu haben mit den Gestaltungen, welche durch Auffassungen im Sinne bestimmter Interessen gesetzt werden ».

54. Ehrenfels, 1890. Sur les qualités de forme chez Ehrenfels et leur parenté dans la philosophie machienne, voir Mulligan et Smith, 1985. 
remarque, nous pouvons très bien considérer que les donations aient un double statut, ontologique et épistémologique, sans que nous ayons pour cela à supposer un autre mode ou instance de conscience qui puisse thématiser cette distinction. C'est ce que Daubert, avec la phénoménologie de Munich, thématisera par les concepts de "sphères d'être ", et c'est en regard de ce type de thématisation que Husserl les accusera plus tard de faire de l'ontologisme $^{55}$. Deuxièmement, les donations recèlent de l'objectif, au sens où elles peuvent contenir des objets identiques saisis sous différents modes, et c'est cet objectif tel quel, tel qu'il est pensé, perçu et non son identité obtenue par réduction, qui est l'objet de la donation. En ce sens, nous pouvons dire que la phénoménologie telle que la conçoit Daubert a une dette envers le positivisme de Mach et d'Avenarius. Troisièmement, la donation, ou la structure de donation, doit être comprise comme une relation de fondation, c'est-àdire que les objectivités que l'on en a abstraites en fondent d'autres dans différentes "sphères ", et la phénoménologie est à comprendre en ce sens comme une théorie des formes et de leurs relations, ou en d'autres termes, comme une théorie des essences. C'est précisément en ce que la théorie des essences n'est qu'une partie de ce que Daubert appelle l' " apercevoir général » que sa conception de la phénoménologie, et non celle de Husserl, peut éviter le rationalisme menant à la réduction en phénoménologie. Quatrièmement, le " fait de la donation des objets » doit être étudié en partant de l'adéquation du donné au saisi, et cette adéquation donne accès aux relations d'essence, ce que Daubert nomme également l'a priori, sensible comme catégorial. Cinquièmement, et cela touche également le problème de l'abstraction, le donné est toujours accessible comme matériel d'une saisie. C'est en en faisant l'extraction, et donc en conservant ce que Daubert appelle les relations de fondation, que peut se pratiquer la phénoménologie. Enfin, la phé-

55. Ce reproche est rapporté dans Spiegelberg, 1982, pp. 171-172. On retrouve un tel reproche, bien qu'on peut dire qu'il vise également Heidegger, dans la Krisis ainsi que dans la postface aux Ideen... : «Ce qui, généralement, cause de grandes difficultés inhérentes à la nature même des choses, c'est la compréhension ou du moins la sûre maîtrise de la différence entre la phénoménologie transcendantale et la psychologie descriptive ou, comme on l'appelle souvent depuis peu, la psychologie phénoménologique ». Plus loin : "Dans les milieux où l'on écarte la réduction phénoménologique comme une originalité sans importance du point de vue philosophique - par quoi on enlève en vérité tout sens à mon œuvre et à ma phénoménologie, ne laissant subsister par suite qu'une psychologie a priori - il arrive assez souvent que précisément cette psychologie qu'on a retenue est identifiée, quant à son sens, avec la psychologie de l'intentionnalité de Franz Brentano ». Hua V/250sq. Traduction française tirée de Husserl, 1993. Dans la Krisis : "Mentionnons d'abord le pire de ces préjugés [contre la phénoménologie transcendantale] : il atteint d'avance la phénoménologie transcendantale en tant que philosophie prétendument déjà disponible, je veux dire que l'on s'imagine d'avance savoir déjà de quoi il s'agit, quelle sorte de philosophie apodictiquement fondée il y a là. Dans le meilleur des cas on a lu mes écrits, ou bien, ce qui est encore plus fréquent, on a pris conseil de mes élèves, dans l'idée qu'ayant reçu mon enseignement ils doivent pouvoir fournir des renseignements dignes de confiance (...) ». Hua VI/520. Traduction française tirée de Husserl, 1976. 
noménologie doit se résoudre à constituer une théorie du donné-même, et éviter toute forme de kantisme ou de platonisme dans la saisie du donné. Ainsi, la phénoménologie ne se fonde ni dans le donné ni dans le saisi, mais elle est une théorie des différentes circulations entre le donné et le saisi, entre les marques ou formes et la nature environnante. Elle se présente ainsi comme une alternative au kantisme car elle n'isole pas l'objet ni ne le déduit par les relations qu'il fonde, ainsi qu'au platonisme car elle ne présuppose pas que l'a priori objectif soit une idéalité et qu'il corresponde à ces formes.

Comme Daubert l'a suggéré dans le dernier manuscrit étudié ici, c'est bien plutôt à partir d'une observation du donné partant d'une attitude scientifique, voire pratique, que celui-ci pourra faire voir les marques ou les formes qui sont incarnées en lui. Comme K. Schuhmann et B. Smith l'ont remarqué : «Daubert (...) holds to a certain primacy of perception. But for him the value of reflection, or of a philosophical theory, is not measured by the degree to which it might overcome perception. The value of reflection depends rather upon its capacity to remain faithful to perception as the source from which it sprang " $"$. Seraient-ce là les véritables sources (Quellen) que Husserl entendait dans son introduction aux Recherches logiques?

\section{Bibliographie}

Des (Hua) Husserliana : Edmund Husserl Gesammelte Werke, éditées sous la responsabilité du Husserl-Archief te Leuven (dir. R. Bernet), aux éditions M. Nijhoff, La Haye à partir de 1950 puis, à partir de 1980, chez Kluwer Academic Publishers, Dordrecht/Boston/Londres :

Hua III : Edmund Husserl, Ideen zu einer reinen Phänomenologie und phänomenologischen Philosophie. Erstes Buch : Allgemeine Einführung

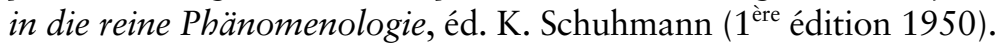

Hua V: Edmund Husserl, Ideen zu einer reinen Phänomenologie und phänomenologischen Philosophie. Drittes Buch : Die Phänomenologie und die Fundamente der Wissenschaften, éd. Marly Biemel, 1952.

Hua VI : Edmund Husserl, Die Krisis der europäischen Wissenschaften und die transzendentale Phänomenologie. Eine Einleitung in die phänomenologische Philosophie, éd. W. Biemel, 1954.

Hua X: Edmund Husserl, Zur Phänomenologie des inneren Zeitbewusstseins (1893-1917), éd. R. Boehm, 1966.

Hua XIII : Edmund Husserl, Zur Phänomenologie der Intersubjektivität. Texte aus dem Nachlass. Erster Teil. 1905-1920, éd. I. Kern, 1973.

Hua XVIII : Edmund Husserl, Logische Untersuchungen. Erster Band: Prolegomena zur reinen Logik. Text der 1. und der 2. Auflage, éd. E. Holenstein, 1975. 
Hua XIX-XX : Edmund Husserl, Logische Untersuchungen. Zweiter Band: Untersuchungen zur Phänomenologie und Theorie der Erkenntnis. En deux volumes, éd. Ursula Panzer, 1984.

\section{Autres sources :}

Avé-Lallemant, E., Die Nachlässe der Münchener Phänomenologen in der Bayerischen Staatsbibliothek, Wiesbaden, Otto Harrassowitz, 1975.

Dummett, M., Les origines de la philosophie analytique, Paris, Gallimard, 1991.

Dummett, M., "Le principe du contexte ", dans Marion, M., Voizard, A., (dir.) Frege. Logique et Philosophie, Montréal, L'Harmattan, 1998.

Ehrenfels, C. von , "Ueber "Gestaltqualitäten" ", Vierteljahrsschrift für wissenschaftliche Philosophie, 14, 1890, pp. 242-92.

Fréchette, G., Husserl, Daubert et la phénoménologie, mémoire, Montréal, Université du Québec à Montréal, 2000.

Fréchette, G., Daubert et la naturalisation de la phénoménologie, communication inédite, Montréal, 2000a.

Frege, G., Les Fondements de l'arithmétique, Paris, Le Seuil, 1969.

Glock, H.-J., A Wittgenstein Dictionary, Oxford, Blackwell, 1996.

Husserl, E., Idées directrices pour une phénoménologie et une philosophie phénoménologique pure, Paris, Gallimard, 1950.

Husserl, E., Recherches logiques, Paris, PUF, 1963.

Husserl, E., La crise des sciences européennes et la phénoménologie transcendantale, Paris, Gallimard, 1976.

Husserl, E., Problèmes fondamentaux de la phénoménologie, Paris, PUF, 1991.

Husserl, E., Idées directrices pour une phénoménologie et une philosophie phénoménologique pures. Livre troisième: la phénoménologie et le fondement des sciences, Paris, PUF, 1993.

Kuhn, H., Avé-Lallemant, E., Gladiator, R. (dir.), Die Münchener Phänomenologie, Den Haag, Martinus Nijhoff, 1975.

Marion, J.-L., Étant donné. Essai d'une phénoménologie de la donation, Paris, PUF, 1997.

Marion, M., Wittgenstein, Finitism, and the Foundations of Mathematics, Oxford, Clarendon Press, 1998.

Mulligan, K., Smith, B., " Mach und Ehrenfels : Über Gestaltqualitäten und das Problem der Abhängigkeit ", dans Fabian R. (dir.), Christian von Ehrenfels. Leben und Werk, Amsterdam, Rodopi, 1985, pp. 85-111.

Schapp, W., Erinnerungen an Edmund Husserl, Wiesbaden, B. Heymann Verlag, 1976. 
Schuhmann, K., «Structuring the Phenomenological Field : Reflections on a Daubert Manuscript ", dans Hamrick, W., Phenomenology in practice and theory, Den Haag, Martinus Nijhoff, 1985.

Schuhmann, K., Smith, B., "Against Idealism : Johannes Daubert vs. Husserl's Ideas I ", Review of Metaphysics, 38, 1985, pp. 763-793.

Schuhmann, K., Smith, B., "Questions : An Essay in Daubertian Phenomenology ", Philosophy and Phenomenological Research, vol. XLVII, no. 3, 1987, pp. 353-384.

Schuhmann, K., "Husserl's Concept of the Noema : A Daubertian critique », Topoi, 8, 1989, pp. 53-61.

Schuhmann, K., Smith, B., " Neo-Kantianism and Phenomenology. The Case of Emil Lask and Johannes Daubert ", Kant-Studien, 1991, pp. 303318

Schuhmann, K., Avé-Lallemant, E., Ein Zeitzeuge über die Anfünge der phänomenologischen Bewegung: Theodor Conrads Bericht aus dem Jahre 1954, Husserl Studies, 9, 1992, pp. 77-90.

Schuhmann, K., Daubert-Chronik, manuscrit inédit, 1996.

Schuhmann, K., "Johannes Daubert als Ästhetiker ", Axiomathes, no. 1-2, 1998, pp. 61-79.

Sepp, H.-R. (dir.), Edmund Husserl und die phänomenologische Bewegung. Zengnisse im Text und Bild, Freiburg/München,Verlag Karl Alber, 1988.

Smid, R. N., "Münchener Phänomenologie - Zur Frühgeschichte des Begriffs », dans Spiegelberg, H., Avé-Lallemant, E., Pfänder-Studien, Den Haag, Martinus Nijhoff, 1982, pp. 109-153.

Smid, R. N., "An Early Interpretation of Husserl's Phenomenology: Daubert and the "Logical Investigations" ", Husserl Studies, 2, 1985, pp. 267-290.

Sommer, M., Husserl und der frühe Positivismus, Frankfurt, Vittorio Klostermann, 1985.

Sommer, M., Evidenz im Augenblick. Eine Phänomenologie der reinen Empfindung, Frankfurt, Suhrkamp, 1987.

Spiegelberg, H., Avé-Lallemant, E., Pfänder - Studien, Den Haag, Martinus Nijhoff, 1982.

Spiegelberg, H., The Phenomenological Movement, Den Haag, Martinus Nijhoff, $3^{\mathrm{e}}$ éd., 1982. 